\title{
Study of Surface Properties of Fused Deposition Modelling Parts
}

\author{
${ }^{1}$ Siddanth Shetty B, ${ }^{2}$ Sunil Gaekwad \\ ${ }^{1}$ M.Tech Student, ${ }^{2}$ Assitant Professor,Department of Mechanical Engineering,The National Institute of Engineering, \\ Mysuru, India.
}

Abstract: Fused Deposition Modeling (FDM) is one of the most commonly used Additive Manufacturing (AM) methods for converting 3D designs into physical models. The printing head melts the thermoplastic material and then extrudes the melted material through the extrusion nozzle layer by layer until the final 3D object is obtained. The build material used here is acrylonitrile butadiene styrene (ABS). The layer by layer addition of the material results in an uneven surface finish of the final product. Studies have shown that the post processing techniques like coating will have a positive impact on the surface finish as well as hardness. In this study, the specimen for the coating processes were printed as per the ASTM standards (ASTM D2240, ASTM D256) needed for mechanical tests. Different post processing techniques involving sputtering, spray painting and acetone dipping were carried out with a negligible change in specimen thickness. The samples were either coated with Copper, or dipped in acetone to smoothen the surface. These coated specimens were then tested for roughness \& hardness and the results showed significant improvements. A comparison has been made between the results of different post processing techniques in order to determine the most suitable process.

Keywords: Additive manufacturing, Fused Deposition Modeling, acrylonitrile butadiene styrene, surface roughness, Rapid Prototyping.

\section{INTRODUCTION}

Additive Manufacturing process are used for making prototypes much quickly and cost effectively. It was developed in the late 1980's by S-Scrott Crum and was later commercialized by Stratasys in 1990's. It is defined as the layer by layer fabrication of three dimensional physical models from CAD files. The application of these ranges from everyday products to aerospace components and medical implants [1]. These processes are classified as liquid, solid and powder based on the build material being used. Here, we use Fused Deposition Modeling machine where thermoplastic filaments are extruded from the heating tip to create the product one layer at a time [2]. However, certain defects arise on FDM parts namely poor surface finish and reduced dimensional accuracy, which remain inherent features generated due to its fundamental working principle. Therefore, different treatment techniques are employed in order to improve the surface properties of the parts printed through FDM.

\section{Literature SURVEY}

A variety of processes have been employed to improve the dimensional accuracy and surface finish of FDM parts. These are classified as pre- \& post processing.

\section{A.Pre-Processing}

The techniques that are used before printing the component, at $\mathrm{CAD}$ model stage, are identified as preprocessing. The optimization of FDM parameters is necessary to achieve higher quality parts [3]. Listed below are some of the significant parameters:

- Build temperature: The temperature at which the material melts and leaves the nozzle.

- Layer thickness: It is the thickness of each layer made to build the part.

- Raster width: The width associated with each raster.

- Orientation: The angle at which specimen is fabricated with respect to build platform [4].

- Raster angle: It refers to the angle of each raster with respect to its previous one.

- $\quad$ Air gap: It is the gap between two neighboring rasters [5].

Some of these pre-processing techniques were employed such as changing in build temperature, layer thickness and build orientation in order to check ideal conditions for printing the part, which would result in improved surface finish, dimensional accuracy and strength. It was seen that at a build temperature of $300^{\circ} \mathrm{C}$, with a layer thickness of $0.254 \mathrm{~mm}$ will provide the best possible output from preprocessing conditions. 


\section{B. Post Processing:}

The techniques that are used after the extrusion of the material from the nozzle, the physical model, are classified as post processing.

\section{i.Sputtering}

Sputtering is usually carried out in argon plasma. The process involves biasing the target negatively which is the source of metal that in turn attracts the argon ions towards the target. The target used here is of copper. The momentum of the argon ions is transferred to the target resulting in the ejection of one or more atoms from the surface of the target [6]. The sputtered atoms, mostly neutral, fly into the plasma and land on the material to be coated. The angular distribution of the sputtered particles follows cosine law. Therefore, like evaporation, sputter deposition occurs essentially along a line-of-sight path with a cosine distribution [7]. The deposition of copper on the ABS material is uneven. The uniformity of the deposited film can be improved by raising the substrate temperature, using a larger target.

\section{ii. Acetone dipping}

Acetone dipping is used as a post processing where parts are dipped in acetone bath for about 30 seconds to get the desired surface finish. The acetone has a good surface peeling characteristics and is used for polishing and finishing the product. After dipping in the acetone solution the increase in weight was noticed [8]. This chemical treatment can remarkably improve the surface finish of ABS prototypes and is a faster, easier and more economical option. A minor reduction of the tensile strength but greater ductility was found in reduced roughness conditions [9]. Nevertheless, immersing ABS in solvent for longer period causes dissolution and there by decreases the volume.

\section{iii. Spray Painting}

The paint process starts with removing all the dirt with the help of clean cloth, and then a thin layer of primer usually polyurethane is applied on the surface. The next comes with spraying another layer of primer in order to obtain bright surface finish; it is then let to dry for an hour. The above process is carried at room temperature. Later the part is sprayed with copper paint. The spraying is done with the help of spray gun held 10 inches apart from the surface being sprayed. The best method of spraying is to work back and forth in straight lines on the surface of the part to produce even lines of paint. Once the painting process is completed, the part is placed in an oven for 24 hours with temperature maintained at $50^{\circ} \mathrm{C}$.

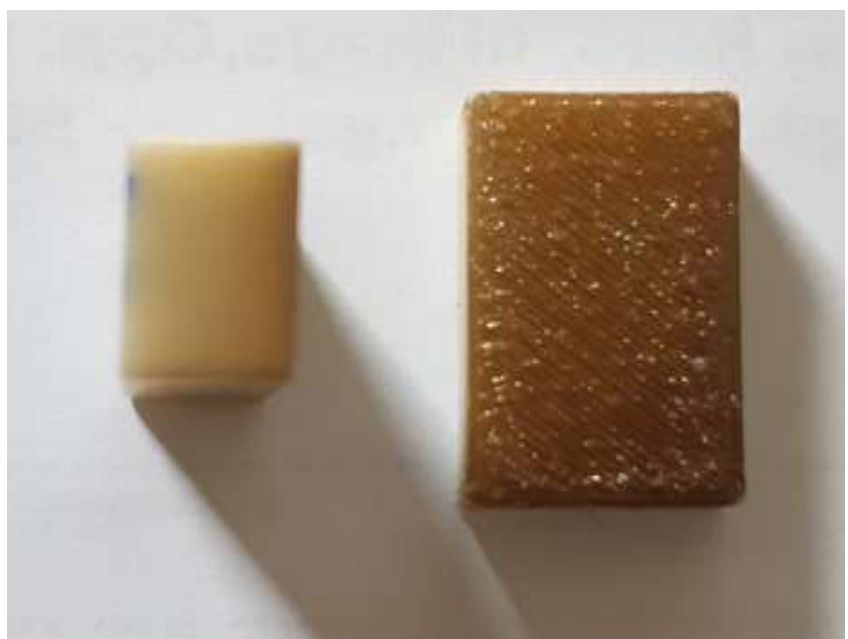

Fig 1. Copper Sputtered ABS Samples
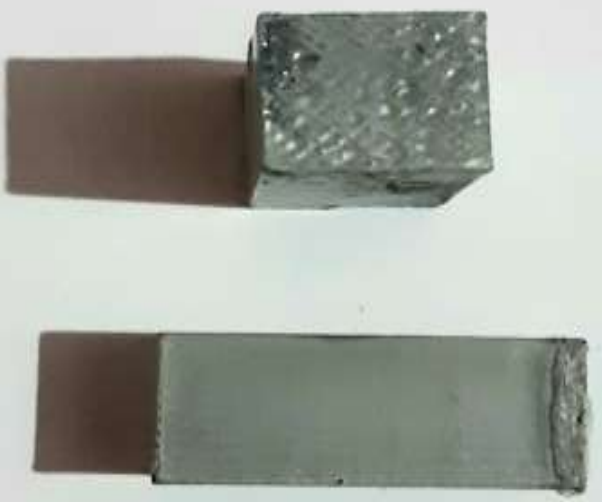

Fig 2. Acetone Dipped ABS Samples

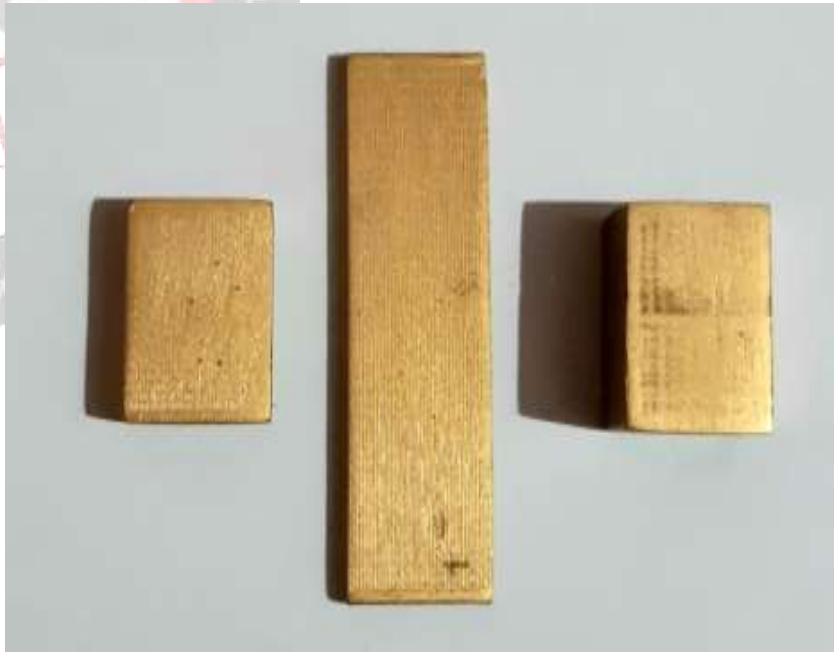

Fig 3. Copper Spray Painted ABS Samples

\section{Testing AND Results}

\section{A. Hardness Test}

The printed parts were tested for hardness as per ASTM D2240 with the help of Durometer [10]. The Shore D scale is used for measuring the hardness. The Durometer intender tip penetration into the sample measures Shore hardness value under the action of the force of a spring [11]. 
Table 1. Hardness Test results of uncoated and coated samples

\begin{tabular}{|c|c|c|c|}
\hline Samples & $\begin{array}{c}\text { Temp } \\
\left({ }^{\circ} \mathbf{C}\right)\end{array}$ & $\begin{array}{c}\text { Layer } \\
\text { Thickness }(\mathbf{m m})\end{array}$ & $\begin{array}{c}\text { Hardness } \\
\text { Value }\end{array}$ \\
\hline Uncoated & 300 & 0.254 & 69.6 \\
\hline Cu Sputtering & 300 & 0.254 & 73.8 \\
\hline Acetone dipping & 300 & 0.254 & 72.4 \\
\hline Spray Painting & 300 & 0.254 & 77.6 \\
\hline
\end{tabular}

Table 1 shows that the hardness value for all the coated samples increases when compared to the uncoated and best result is obtained in spray painting process.

\section{B. Roughness Test}

The printed samples were measured for roughness with help of Atomic Force Microscopy (AFM) [12]. It is also known by scanning force microscopy (SFM). AFM is one of the most preferred instruments that are used to study the surface smoothness of a component. It uses a very high resolution of scanning probe microscopy (SPM) on the order of fractions of nanometer. On the nanometer scale it is one of the most important tools for imaging the specimen. A cantilever with a sharp probe is used so that it can scan the surface of the specimen. According to Hooke's law, the force between the sample and tip deflects the cantilever when the tip of the probe travels near to a surface.

Table 2: Roughness test results for uncoated and coated samples

\begin{tabular}{|c|c|c|c|}
\hline Samples & Temp $\left({ }^{\circ} \mathrm{C}\right)$ & $\begin{array}{c}\text { Layer Thickness } \\
(\mathrm{mm})\end{array}$ & $\mathrm{R}_{\mathrm{a}}(\mathrm{nm})$ \\
\hline Uncoated & 300 & 0.254 & 69.828 \\
\hline Cu Sputtering & 300 & 0.254 & 65.812 \\
\hline $\begin{array}{c}\text { Acetone } \\
\text { Dipping }\end{array}$ & 300 & 0.254 & 54.866 \\
\hline $\begin{array}{c}\text { Cu Spray } \\
\text { Painting }\end{array}$ & 300 & 0.254 & 46.028 \\
\hline
\end{tabular}

Table 2 shows that the roughness value for all the coated samples decreases when compared to the uncoated and best result is obtained in spray painting process.

$\mathrm{R}_{\mathrm{a}}$ : Roughness Average - It is defined as the arithmetic average of the absolute values of the profile heights over the evaluated length.
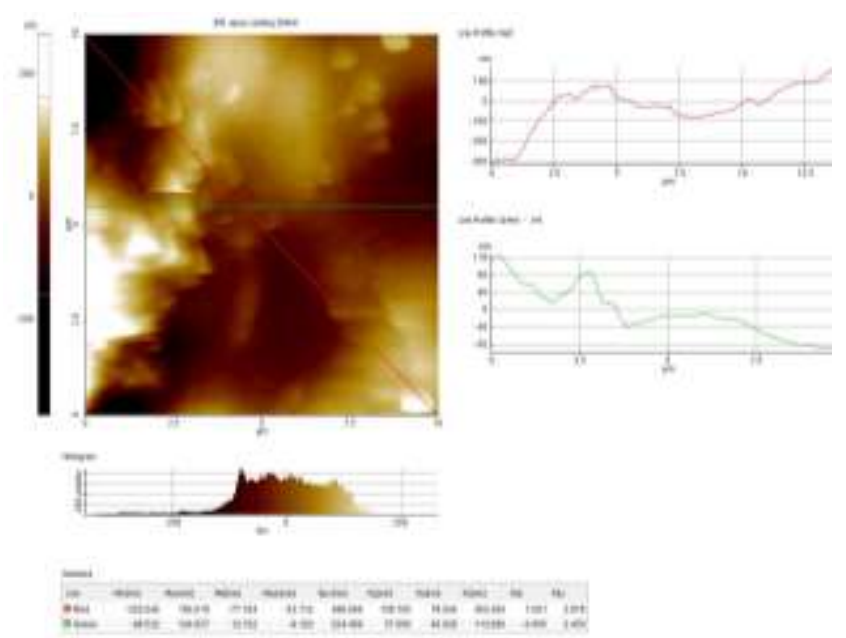

Fig 4. Topography of copper spray painted ABS sample imaged using AFM

The graph above represents the surface roughness of the copper spray painted sample in horizontal and diagonal direction along the sample surface. The green line in the graph refers to roughness along the horizontal axis and the red line refers to the roughness along diagonal axis.

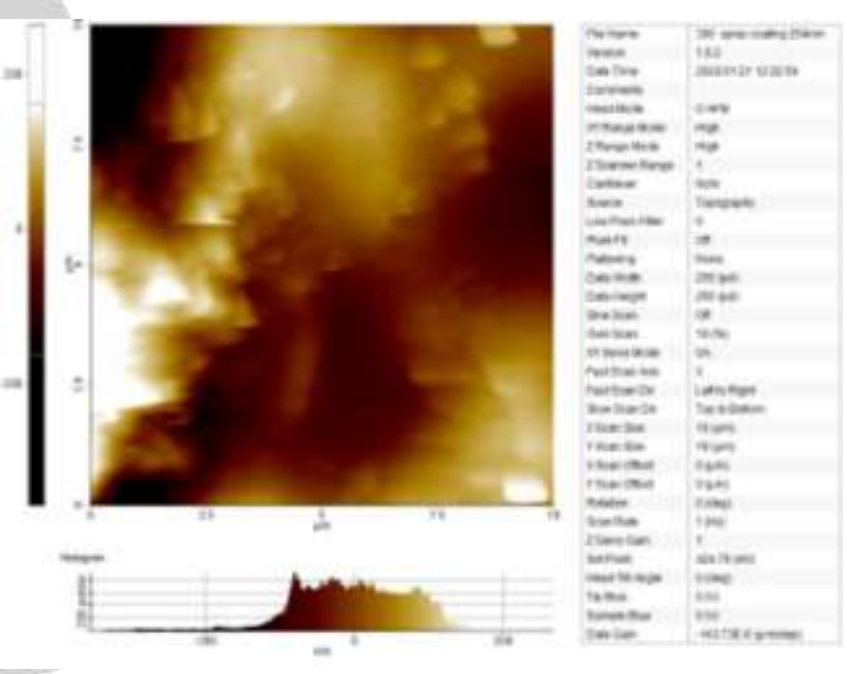

Fig 5. Information regarding the copper spray painted ABS sample

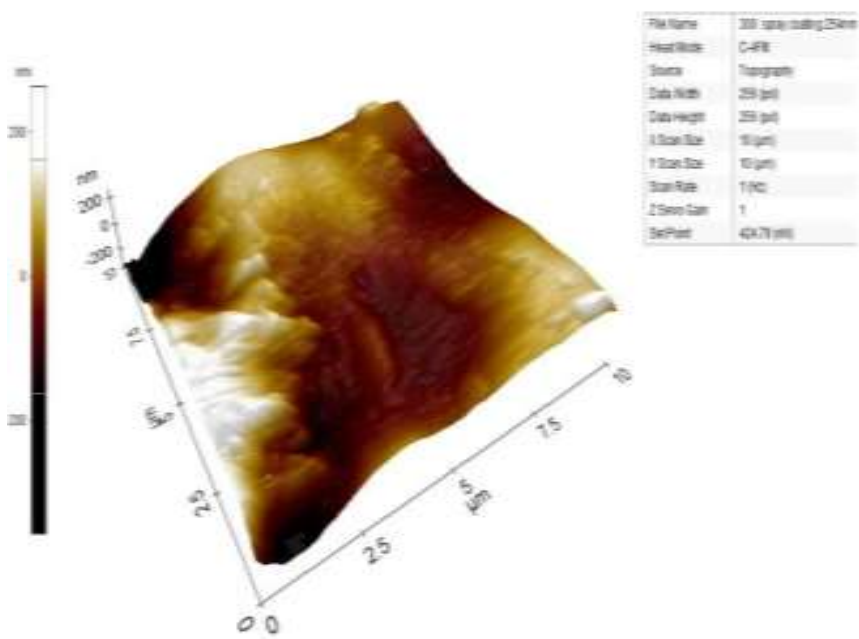

Fig 6. 3D image of Copper spray painted ABS sample 


\section{Conclusion}

In this study, the samples printed at build temperature of $300^{\circ} \mathrm{C}$ and $0.254 \mathrm{~mm}$ layer thickness were chosen to provide best possible output and 3 types of coating namely sputtering, acetone dipping, copper spray painting were done on samples and tested for hardness and roughness.

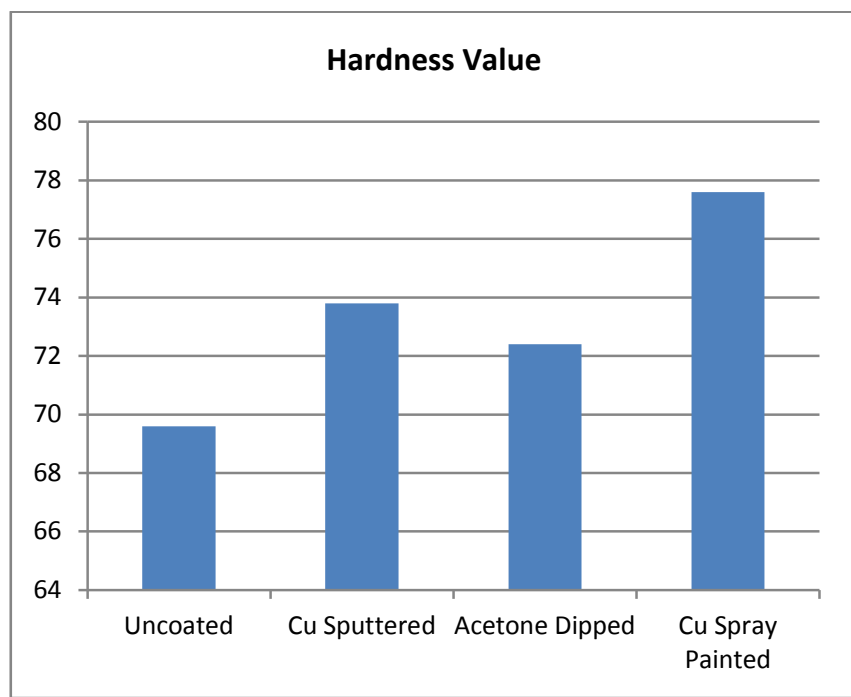

Fig 5. Chart showing value of hardness for different post process

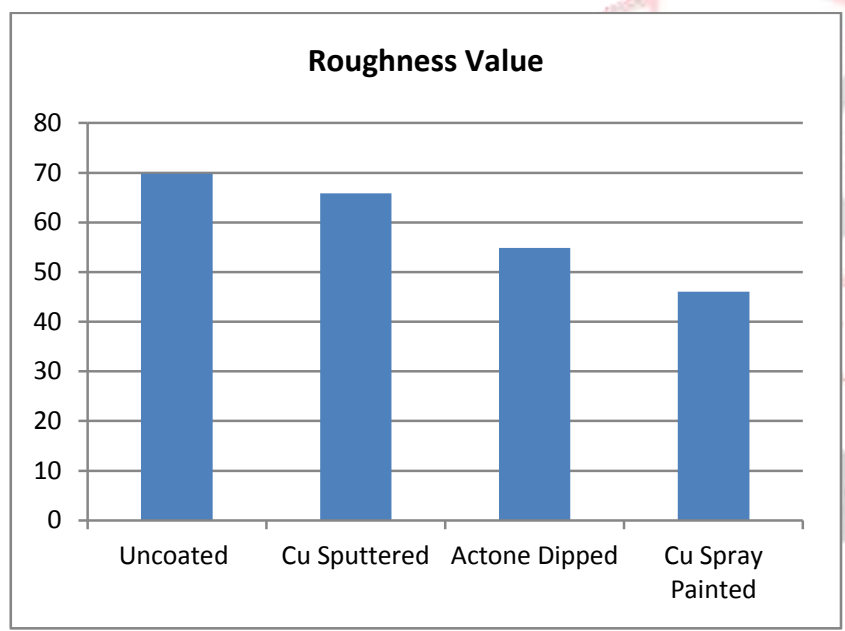

Fig 6. Chart Showing roughness of different post processes

The results showed improvement in both hardness and roughness value for all the coating processes when compared with uncoated samples. Therefore, it is clear that coating the samples improve the surface properties of the printed parts. The best outcome was obtained in samples that had undergone spray painting process with increased hardness and decreasing surface roughness.

\section{REFERENCES}

[1] Kamaljit Singh Boparai, Rupinder Singh \& Harwinder Singh; Development of rapid tooling using fused deposition modeling: A review; Rapid Prototyping Journal, March 2016, DOI: 10.1108/RPJ04-2014-0048.
[2] S Dhaneshmand, C Aghanjafi \& A Ahmadi Nadooshan; The effect of chromium coating on RP technology for air foil manufacturing; Sadhana, Vol 35, Part 5, October 2010, pp. 569-584, Indian academy of Sciences.

[3] Ravi Patel, Satyam Patel, Jaimin Patel "A Review on Optimization of Process Parameter of Fused Deposition Modeling for etter Dimensional Accuracy" International Journal of Engineering Development and Research 2014 IJEDR, Volume 2, Issue 2, ISSN: 2321- 9939.

[4] M. S. Khan and J. P. Dash (2019), Enhancing surface finish of fused deposition of modeling parts, https://doi.org/10.1007/978-981-13-0305-0_5.

[5] Chil-Chyuan Kuo, Shenj-Jie Su "A simple method for improving surface quality of rapid prototype" Indian Journal of Engineering and Material Science, Vol20, December 2013, (pp. 465-470).

[6] Hand Book of Deposition Technologies for Films and Coating (Third Edition), 2010, (pp. 253-296), https://doi.org/10.1016/B978-0-8155-2031-3.000053.

[7] Yu. V. Martynenko, A. V. Rogav and V. I. Shul'ga Angular Distribution of Atoms during the Magnetron Sputtering of polycrystalline targets, July 18, 2011, DOI: $10.1134 / \mathrm{S} 1063784212040196$.

[8] Kwang-Ho Jo, Yeon-Seong Jeong, Jae-Hak Lee, and Seok-Hee Lee, A Study of Post Processing Methods for Improving the Tightness of a Part Fabricated by Fused Deposition Modeling, International Journal of Precision Engineering and Manufacturing, Vol. 17, No. 11, (pp. 1541-1546), DOI: 10.1007/s12541-0160180-z, November 2016.

[9] L.M. Galantucci, F. Lavecchia, G. Percoco, Quantitative analysis of a chemical treatment to reduce roughness of parts fabricated using fused deposition modeling, CIRP Annals - Manufacturing Technology, Volume 59, Issue 1, 2010, Pages 247250, ISSN 0007-8506.

[10] ASTM D2240-15, Standard Test Method for Rubber Property Durometer Hardness, ASTM International, West Conshohocken, PA, 2015, www.astm.org.

[11] Jaspreet Singh, Rupinder Singh, Harwinder Singh, Experimental Investigation for shore hardness of ABS replicas fabricated by fused deposition modeling and vapor smoothing process, International journal of advanced multidisciplinary research, Vol 9(1), 2017, (pp. 29-34).

[12] Kovan, V., Altan, G., Topal, E. S., \& Camurlu, H. E. (2016, February). Surface roughness effect on the 3D printed butt joints strength. International Scientific Conference BALTTRIB (pp. 117-121). 